\title{
A Modified Implicit Z-Matrix Method for Power Flow Study of Advanced Distribution Systems
}

\author{
Non Member \\ Member \\ Zheng Yan \\ (Ibaraki University) \\ Non Member \\ Koichi Nara \\ (Ibaraki University) \\ Hongyu Zhou \\ (Tsinghua University)
}

\begin{abstract}
This paper studies the power flow computation for advanced distribution systems where multiple PV-buses appear. The background for this study is that with the introduction of various dispersed generation sources into modern and future distribution systems, it may be necessary to treat multiple PV-buses in distribution power flow calculations. Meanwhile, in analysing and planning such complex systems, hundreds or thousands of power flow computations are required. Therefore efficiency of the power flow method is of vital importance for reducing the computing burden. The method presented in this paper is a modification to conventional implicit $Z$ matrix method by which only one voltage specified bus can be dealt with efficiently. The modification is based on direct mathematical derivation and therefore overcomes drawbacks of heuristics based techniques. The method tries to solve for the total unknown variables simultaneously without use of trial and error iterations. Computational experience shows that the method is especially suitable for power flow study of distribution systems. Comparisons with Newton power flow method are provided to illustrate the efficiency of the method.
\end{abstract}

Keywords: Distribution Power Flow, Implicit Z-Matrix Method, Sparse Matrix/Vector Techniques

\section{Introduction}

Power flow study has been widely used in expansion planning of future power systems as well as in determination of the best operation of existing systems. There are many kinds of power flow computation methods [1]: bus admittance iterative method, bus impedance (or $Z$ ) matrix method, Newton's method [2] and Fast Decoupled method [3]. Among which, Newton's method has found most wide applications in virtue of its flexibility, robustness and quadratic convergence. In contrary to the general applicability of Newton's method, in some special cases, other methods may exhibit better computational efficiency and robustness. For example, Fast Decoupled Power Flow (FDPF) method is generally faster for well conditioned systems and exhibit superlinear convergence. But its convergence becomes poor if branches of high $|r / x|$ ratios exist in the systems. While for distribution systems with one voltage specified bus, implicit $Z$ matrix method exhibits faster computing speed.

Recently, more and more attentions have been paid to distribution power flow computations by thorough utilization of the radial or sparsely meshed structure of the systems [4][6]. In conventional distribution power flow computation, it is generally assumed that the slack bus is the only one voltage specified bus in the whole system. In reference [4], a forward/backward sweep method was designed for systems with radial structure. The method is a direct application of Kirchhoff's laws, therefore it's mathematically equivalent to the implicit $Z$ matrix method, but with much more enhancement in algorithmic implementation. The method suffers from deficiency when several voltage specified buses exist in the analysed systems.
The forward/backward sweep method has also been the kernel algorithm of referceces [5] and [6]. Further consideration was paid to solving three phase distribution power flow in [6]. In which, loops are split into radial feeders by introducing breaking points which results in a dense matrix equation with dimension equaling to number of loops. PV buses are dealt with by a dense sensitivity matrix with dimension equaling to number of PV buses. The method is not appropriate for those distribution systems where there may exist many loops in the network.

However, for modern and future advanced distribution systems, except the slack bus, there may exist multiple PV-buses for the reason of the introduction of various dispersed generating units. The systems may also contain many power delivery loops for recognition of its flexible and reliable functions. One example of such systems is the socalled FRIENDS (Flexible, Reliable, Intelligent and Energy coNservative Distribution Systems) discussed in the litera ture [7]. This paper is addressed to the power flow computations of these kinds of advanced distribution systems.

In a flexible power delivery system such as FRIENDS, many advanced functionalities may be encompassed. For optimal structure analysis and planning of such a complex system, numerous power flow calculations are required. Therefore the excellent performance of the power flow program will certainly provide a good starting point of study. In the process of studying the system, minor network operations with a few branches' deletion or addition occur frequently, the system structure changes a little for each network operation. Newton's method cannot attain its maximum efficiency for this case because it executes every power flow computation from scratch. While decoupled method is not suitable 
to distribution systems where branches with wide range of $|r / x|$ ratios exist. With such research background, the implicit $Z$ matrix method is chosen in this paper, because it involves trivial partial refactorization or factors updating of admittance matrix for each network operation.

The proposed method is a natural extension of the traditional implicit $Z$ matrix method. The modification takes full advantage of the special structure of the studied system, with special treatments with the voltage specified equalities and reactive power outputs. No special care is needed for delivery loops, and the reactive power outputs of PV-buses can be solved simultaneously with bus voltages in an unified iterative process, therefore it overcomes the drawbacks of conventional sensitivity-based techniques [6]. Numerical experience indicates that the proposed method is very effective. Comparisons with Newton's method are also reported.

\section{Brief Description of Implicit $Z$ Matrix} Method

\subsection{Basic Formulation of Power Flow Problem}

The power flow problem can be viewed as a set of nonlinear equations that are derived from network nodal equations. For a system containing $N$ buses, after applying the Kirchhoff's laws, the network equation can be written as:

$$
Y V=I
$$

where $Y$ is the $N \times N$ symmetrical and sparse bus admittance matrix, $V$ and $I$ are $N$-vectors of bus voltages and injected currents, respectively. Generally, the injected currents are nonlinear functions of the unknown voltages, therefore Eq.(1) can only be solved by iterative method. Expressions for individual quantities in Eq.(1) are as follows:

$$
\begin{gathered}
Y_{i j}=G_{i j}+j B_{i j} \\
V_{i}=e_{i}+j f_{i} \\
I_{i}=I_{P_{i}}+j I_{Q_{i}}=\left(S_{i} / V_{i}\right)^{*} \\
(i=1, \cdots, N \quad j=1, \cdots, N)
\end{gathered}
$$

where $G_{i j}$ and $B_{i j}$ are real and imaginary parts of admittance matrix element $Y_{i j} ; e_{i}$ and $f_{i}$ are real and imaginary parts of voltage $V_{i} ; P_{i}$ and $Q_{i}$ are real and reactive parts of apparent power $S_{i}$ injected at bus $i, I_{P i}$ and $I_{Q i}$ are real and reactive parts of the injected current $I_{i}$ at bus $i$. Eq.(1) is a equation in complex numbers, it can also be transformed into the form expressed in real numbers. Substitute Eq.(2) into Eq.(1), we have

$$
\begin{aligned}
\sum_{j=1}^{N}\left(B_{i j} e_{j}+G_{i j} f_{j}\right) & =I_{Q i}=\left[P_{i} f_{i}-Q_{i} e_{i}\right] /\left|V_{i}\right|^{2} \\
\sum_{j=1}^{N}\left(G_{i j} e_{j}-B_{i j} f_{j}\right) & =I_{P i}=\left[P_{i} e_{i}+Q_{i} f_{i}\right] /\left|V_{i}\right|^{2} \\
(i & =1, \cdots, N)
\end{aligned}
$$

where $\left|V_{i}\right|=\sqrt{e_{i}^{2}+f_{i}^{2}}$ is the magnitude of $V_{i}$. Eq.(3) can be arranged in matrix form:

$$
\overline{Y V}=\bar{I}
$$

Here $\bar{Y}$ is a $2 N \times 2 N$ real symmetrical matrix, it has the same sparsity structure as $Y$ but with $2 \times 2$ block elements, its $(i, j)$ block element $\bar{Y}_{i j}$ is of the form:

$$
\bar{Y}_{i j}=\left[\begin{array}{cc}
B_{i j} & G_{i j} \\
G_{i j} & -B_{i j}
\end{array}\right]
$$

$\bar{V}$ and $\bar{I}$ are $2 N$-vectors with $N$ block entries, the $i$-th entry of $\bar{V}$ is of the form:

$$
\overline{V_{i}}=\left[e_{i}, f_{i}\right]^{T}
$$

and the $i-$ th entry of $\bar{I}$ is of the form:

$$
\bar{I}_{i}=\left[I_{Q i}, I_{P_{i}}\right]^{T}
$$

Eq.(1) and Eq.(4) are mathematically equivalent, and if proper programming techniques are used, they are also equivalent in computational efficiency.

It should be noted that for power flow computation, the voltage magnitude and angle of slack bus are specified, so the equation corresponding to slack bus should be deleted from Eq.(1) or Eq.(4). However, for convenience of presentation, the equation for slack bus is still preserved in the following discussion. Algorithmically, large numbers can be inserted in to the relevant diagonals of $Y$ or $\bar{Y}$ to make the associated equations dummy. Programming design with this technique is trivial and has also been proven effective and successful. For deriving the improved method proposed in this paper, Eq.(3) or Eq.(4) should be used instead of Eq.(1). Therefore Eq.(3) and Eq.(4) are the basic formulations of the power flow problem discussed in this paper.

\subsection{Implicit $Z$ Matrix Method}

Numerous power flow methods can be derived from Eq.(1) or Eq.(4), with their final purpose of determining the unknown bus voltage vector $V$ and therefore the operating state of the system. In the traditional explicit Z-matrix method [8], for each estimated bus injected current vector $I$, an improved bus voltage vector $V$ is calculated by using inverse of matrix $Y$, i.e. the full bus impedance matrix $Z$ :

$$
V=Y^{-1} I=Z I
$$

Because of the required computer memory storage and great computing burden, explicit $Z$-matrix method is rarely used in modern application. On the other hand, implicit $Z$-matrix method need not to evaluate the inverse of $Y$, it only performs triangular factorization for $Y$ in $\mathrm{Eq}$. (1) or $\bar{Y}$ in Eq.(4). We concentrate on the case of Eq.(4), in which only calculations with real numbers are involved in factorization of $\bar{Y}$ :

$$
\bar{Y}=L D U=L D L^{T}
$$

where $L$ is a lower triangular matrix, and $D$ is a diagonal matrix. Factorization preserves the sparsity of $\bar{Y}$, and the factors are repeatedly used in forward and backward substitutions in the iterative process of solving $\mathrm{Eq}$.(4) with great saving in computation per iteration, therefore implicit $Z$ matrix method is much faster than direct $Z$-matrix method.

\subsection{Drawback of Implicit $Z$ Matrix Method}

In each iteration of the Implicit $Z$ matrix method, estimated voltage vector $\bar{V}$ is substituted into the right-handside of Eq.(4) to get an estimated current vector $\bar{I}$, and then the improved bus voltage vector can be obtained by solving Eq.(4), using the available factors of Eq.(6). Convergence is reached if variations of voltages between two adjacent iterations are small enough. The method is most suitable to systems with only one slack bus. For systems with multiple voltage specified buses (or PV-buses), the convergence tends to be poor. The reason is that the information that the magnitude of each PV bus is set to be fixed has not been 
utilized. The process doesn't distinguish the difference between a PV-bus and a PQ-bus. Therefore in modern power system computation, application of the implicit $Z$ matrix method is only confined to distribution systems, where only the voltage of the slack bus is specified. For dealing with the case of multiple PV-buses, heuristic-based techniques have to be used to update the reactive powers of PV-buses, and the method generally takes much more iterations to converge.

2.4 Convergence Analysis of Implicit $Z$ Matrix Method

Strict analysis of the convergence property of Implicit $Z$ matrix method is not a simple task. In this section a brief and simplified convergence analysis is given to help to understand the mechanism and applicable conditions. For convenience of analysis, Eq.(1) will be used and polar coordinate expressions for voltages are adopted. Suppose the exact solution of the bus voltage vector is $V$, and the corresponding bus injected current vector is $I$. For the $(k+1)$-th iteration, the approximated solution of bus voltage is $V^{(k+1)}$ which is calculated by solving the following equation:

$$
Y V^{(k+1)}=I^{(k)}
$$

where the bus injected current $I^{(k)}$ is estimated by $V^{(k)}$ of $k$-th iteration. Subtracting Eq.(1) from the two sides of the above equation, we get:

$$
\Delta V^{(k+1)}=V^{(k+1)}-V=Y^{-1}\left(I^{(k)}-I\right)=Z \Delta I^{(k)}
$$

The $i-$ th element of the vector $\Delta V^{(k)}$ is defined as:

$$
\Delta V_{i}^{(k)}=V_{i}^{(k)}-V_{i}=\left|V_{i}^{(k)}\right| \angle \theta_{i}^{(k)}-\left|V_{i}\right| \angle \theta_{i}=\left|\Delta V_{i}^{(k)}\right| \angle \delta_{i}^{(k)}
$$

where

$$
\left|\Delta V_{i}^{(k)}\right|=\sqrt{\left|V_{i}^{(k)}\right|^{2}+\left|V_{i}\right|^{2}-2\left|V_{i}^{(k)}\right|\left|V_{i}\right| \cos \left(\theta_{i}^{(k)}-\theta_{i}\right)}
$$

and

$$
\begin{aligned}
\delta_{i}^{(k)} & =\arcsin \frac{\left|V_{i}^{(k)}\right| \sin \theta_{i}^{(k)}-\left|V_{i}\right| \sin \theta_{i}}{\left|\Delta V_{i}^{(k)}\right|} \\
& =\arccos \frac{\left|V_{i}^{(k)}\right| \cos \theta_{i}^{(k)}-\left|V_{i}\right| \cos \theta_{i}}{\left|\Delta V_{i}^{(k)}\right|}
\end{aligned}
$$

here $\left|V_{i}\right|$ and $\theta_{i}$ are magnitude and angle of voltage $V_{i}$, respectively, and $\angle \theta_{i}=\cos \theta_{i}+j \sin \theta_{i}$. And the $i$-th element of $\Delta I^{(k)}$ can be calculated as follows:

$$
\begin{aligned}
\Delta I_{i}^{(k)} & =\left(S_{i} / V_{i}^{(k)}\right)^{*}-\left(S_{i} / V_{i}\right)^{*} \\
& =\left(P_{i}-j Q_{i}\right)\left(\frac{\angle \theta_{i}^{(k)}}{\left|V_{i}^{(k)}\right|}-\frac{\angle \theta_{i}}{\left|V_{i}\right|}\right) \\
& =\left(P_{i}-j Q_{i}\right) \frac{\Delta w_{i}^{(k)}}{\left|V_{i}^{(k)}\right|\left|V_{i}\right|}
\end{aligned}
$$

where $\Delta w_{i}^{(k)}$ is defined as:

$$
\Delta w_{i}^{(k)}=\left|V_{i}\right| \angle \theta_{i}^{(k)}-\left|V_{i}^{(k)}\right| \angle \theta_{i}=\left|\Delta w_{i}^{(k)}\right| \angle \psi_{i}^{(k)}
$$

And it can be proved that:

$$
\left|\Delta w_{i}^{(k)}\right|=\left|\Delta V_{i}^{(k)}\right|
$$

and

$$
\begin{aligned}
\psi_{i}^{(k)} & =\arcsin \frac{\left|V_{i}\right| \sin \theta_{i}^{(k)}-\left|V_{i}^{(k)}\right| \sin \theta_{i}}{\left|\Delta V_{i}^{(k)}\right|} \\
& =\arccos \frac{\left|V_{i}\right| \cos \theta_{i}^{(k)}-\left|V_{i}^{(k)}\right| \cos \theta_{i}}{\left|\Delta V_{i}^{(k)}\right|}
\end{aligned}
$$

Therefore we have:

$$
\Delta w_{i}^{(k)}=\left|\Delta V_{i}^{(k)}\right| \angle \delta_{i}^{(k)} \angle\left(\psi_{i}^{(k)}-\delta_{i}^{(k)}\right)=\Delta V_{i}^{(k)} \angle \phi_{i}^{(k)}
$$

where

$$
\phi_{i}^{(k)}=\psi_{i}^{(k)}-\delta_{i}^{(k)}
$$

Substitute the above result into Eq.(9), we obtain:

$$
\Delta I_{i}^{(k)}=\left(P_{i}-j Q_{i}\right) \frac{L \phi_{i}^{(k)}}{\left|V_{i}^{(k)}\right|\left|V_{i}\right|} \Delta V_{i}^{(k)} \quad(i=1, \cdots, N)
$$

Define the following two $N \times N$ diagonal matrices:

$$
\begin{gathered}
S=\operatorname{diag}\left[P_{1}-j Q_{1}, \cdots, P_{N}-j Q_{N}\right] \\
C^{(k)}=\operatorname{diag}\left[\frac{L \phi_{1}^{(k)}}{\left|V_{1}^{(k)}\right|\left|V_{1}\right|}, \cdots, \frac{L \phi_{N}^{(k)}}{\left|V_{N}^{(k)}\right|\left|V_{N}\right|}\right]
\end{gathered}
$$

Then we have the matrix form of Eq.(10):

$$
\Delta I^{(k)}=S C^{(k)} \Delta V^{(k)}
$$

Substitute the above equation into the Eq.(8), finally we obtain the following relationship:

$$
\begin{aligned}
\Delta V^{(k+1)} & =Z S C^{(k)} \Delta V^{(k)} \\
& =\left(Z S C^{(k)}\right)\left(Z S C^{(k-1)}\right) \cdots\left(Z S C^{(0)}\right) \Delta V^{(0)}
\end{aligned}
$$

Taking 2-norms in the two sides of the above equation, we get the following inequality:

$$
\begin{aligned}
\left\|\Delta V^{(k+1)}\right\|_{2} & \leq \prod_{t=0}^{k}\left(\|Z S\|_{2}\left\|C^{(t)}\right\|_{2}\right)\left\|\Delta V^{(0)}\right\|_{2} \\
& =\|Z S\|_{2}^{k+1} \prod_{t=0}^{k}\left\|C^{(t)}\right\|_{2}\left\|\Delta V^{(0)}\right\|_{2}
\end{aligned}
$$

With the increase of the iteration number $k,\left\|\Delta V^{(k+1)}\right\|_{2}$ must tend to zero in order to get convergence. This requirement can be attained if the right-hand-side of Eq.(12) approaches to zero with the increase of $k$. Because

$$
\begin{aligned}
\left\|C^{(t)}\right\|_{2} & =\max \left\{\frac{\left|\angle \phi_{i}^{(t)}\right|}{\left|V_{i}^{(t)}\right|\left|V_{i}\right|}, i=1, \cdots, N\right\} \\
& \approx \max \left\{\frac{1}{\left|V_{i}\right|^{2}}, i=1, \cdots, N\right\}
\end{aligned}
$$

is an upper bounded nonzero number, $\left\|C^{(t)}\right\|_{2}$ can be approximatively treated as a constant for limited iteration numbers. Hence the degree of magnitude of the right-hand-side of Eq.(12) depends on matrix norm $\|Z S\|_{2}$. Therefore for convergence of the implicit $Z$ matrix method, the following sufficient condition can be established:

$$
\rho(Z S) \leq\|Z S\|_{2} \leq\|Z\|_{2}\|S\|_{2}=\rho(Z) \rho(S)<1
$$

here $\rho(\cdot)$ is the spectral radius of the relevant matrix. The spectral radius of an $N \times N$ matrix $X$ is defined as the largest 
absolute value among the $N$ eigenvalues $\left\{\lambda_{i}, i=1, \cdots, N\right\}$ of $X$, i.e.

$$
\rho(X)=\max \left\{\left|\lambda_{1}\right|,\left|\lambda_{2}\right|, \cdots,\left|\lambda_{N}\right|\right\}
$$

The above sufficient condition means that the convergence of implicit $Z$ matrix method mainly depends on spectral radius of matrix $Z=Y^{-1}$ and the load level of the system (i.e. the diagonal terms of matrix $S$ ). The smaller the spectral radius of $Z$ is and the lower the load level is, the better the convergence will be. Because the spectral radius of $Z$ is a network inherent parameter which cannot be changed by computational method itself, the implicit $Z$ matrix method is generally recommended to power flow computation of systems with relatively lower loads, especially distribution systems. Convergence can be improved by including parts of loads into $Y$ matrix to reduce diagonal terms of $S$.

\section{Improved Implicit $Z$ Matrix Method}

In research of future distribution system, the network structure will be more complex than present distribution system, and it may contain several PV-buses and may have large number of power delivery loops. In this case, the traditional implicit Z-matrix method must be modified while preserving its original simplicity.

Temporarily suppose that the system contains one PV-bus only at bus $m$. We have $P_{G m}=P_{m}^{S P}$ and $\left|V_{m}\right|=\left|V_{m}\right|^{S P}$. The net injected real and reactive powers at bus $m$ are $P_{m}=$ $P_{G m}-P_{L m}$ and $Q_{m}=Q_{G m}-Q_{L m}$. Here subscripts $G$ and $L$ represents generation and load, respectively. Accurate $Q_{G m}$ is not available before convergence of power flow calculation. From Eq.(2) and Eq.(3), the reactive and active parts of the current injected into bus $m$ can be evaluated as:

$$
\begin{aligned}
& I_{Q m}=\left[\left(P_{G m}-P_{L m}\right) f_{m}-\left(Q_{G m}-Q_{L m}\right) \epsilon_{m}\right] /\left|V_{m}\right|^{2} \\
& I_{P m}=\left[\left(P_{G m}-P_{L m}\right) e_{m}+\left(Q_{G m}-Q_{L m}\right) f_{m}\right] /\left|V_{m}\right|^{2}
\end{aligned}
$$

It can be seen that the specified real power generation $P_{G m}^{S P}$ is naturally satisfied by substituting it into the above equation, but the specified bus voltage $\left|V_{m}\right|^{S P}$ cannot be satisfied because the equality $\left|V_{m}\right|^{S P}=\sqrt{e_{m}^{2}+f_{m}^{2}}$ is never applied in the calculation. Traditional method tries to modify the reactive power generation $Q_{G m}$ in order that $e_{m}$ and $f_{m}$ may indirectly converge to their true values. There exist not general rules for modifying $Q_{G m}$, and the modification is heuristic based and only applied after each iteration, this is the reason why generally the traditional method's convergence becomes poor for systems with multiple PV buses.

In this paper, we treat the reactive power $Q_{G m}$ as an unknown variable. For an estimated $Q_{G m}$, its increment $\Delta Q_{G m}$ will be solved simultaneously with the unknown voltages. Using ${ }^{c}$ to denote superscript of current values. Considering the power flow equation corresponding to bus $m$, replacing $Q_{G m}$ by $Q_{G m}^{c}+\Delta Q_{G m}$ and moving terms related to $\Delta Q_{G m}$ to left-hand-side, the $m$-th power flow equation in Eq.(3) can be written as:

$$
\begin{aligned}
& \sum_{j=1}^{N}\left(B_{m j} e_{j}+G_{m j} f_{j}\right)+\frac{e_{m}^{c}}{\left|V_{m}\right|^{2}} \Delta Q_{G m}=I_{Q m} \\
& \sum_{j=1}^{N}\left(G_{m j} e_{j}-B_{m j} f_{j}\right)-\frac{f_{m}^{c}}{\left|V_{m}\right|^{2}} \Delta Q_{G m}=I_{P_{m}}
\end{aligned}
$$

where $e_{m}^{c}$ and $f_{m}^{c}$ are current values of $e_{m}$ and $f_{m}$, and $I_{Q m}$ and $I_{P m}$ are calculated by $e_{m}^{c}$ and $f_{m}^{c}$.
Because of the introduction of the unknown $\Delta Q_{G m}$, a new equation is required in order that the total unknowns are equal to the total equations. A natural choice of such a new equation is the voltage specified equation $\left|V_{m}\right|^{2}=e_{m}^{2}+f_{m}^{2}$ for PV-bus $m$. Applying the Newton's method to the voltage specified equation around the current iterate, we can get:

$$
e_{m}^{c} \Delta e_{m}+f_{m}^{c} \Delta f_{m}=\frac{1}{2}\left(\left|V_{m}\right|^{2}-e_{m}^{c 2}-f_{m}^{c 2}\right)
$$

Substitute $\Delta e_{m}=e_{m}-e_{m}^{c}$ and $\Delta f_{m}=f_{m}-f_{m}^{c}$ into the above equation, we have:

$$
e_{m}^{c} e_{m}+f_{m}^{c} f_{m}=\frac{1}{2}\left(\left|V_{m}\right|^{2}+e_{m}^{c 2}+f_{m}^{c 2}\right)=U_{G m}
$$

With the above treatment of PV-bus $m$, and by incorporating Eq.(13) and Eq.(15) into Eq.(3), Eq.(4) is expanded as:

$$
\left[\begin{array}{cc}
\bar{Y} & E_{Q m} \\
E_{V m}^{T} & 0
\end{array}\right]\left[\begin{array}{c}
\bar{V} \\
q_{G m}
\end{array}\right]=\left[\begin{array}{c}
\bar{I} \\
U_{G m}
\end{array}\right]
$$

where $q_{G m}=\Delta Q_{G m} /\left|V_{m}\right|^{2}$ is an auxiliary variable to replace $\Delta Q_{G m}$, and $E_{Q m}$ and $E_{V m}$ are $2 N$ sparse vectors of $N$ block entries, in which only their $m$-th block entries are non-zeroes, i.e.:

$$
\begin{aligned}
E_{Q m} & =\left[0,0, \cdots, e_{m}^{c},-f_{m}^{c}, \cdots, 0,0\right]^{T} \\
E_{V m} & =\left[0,0, \cdots, e_{m}^{c}, f_{m}^{c}, \cdots, 0,0\right]^{T}
\end{aligned}
$$

For the general case where there are $M$ PV-buses in the system, similar straightforward treatment can be applied. Then the above formulation can be expanded as:

$$
\left[\begin{array}{cc}
\bar{Y} & E_{Q} \\
E_{V}^{T} & 0
\end{array}\right]\left[\begin{array}{c}
\bar{V} \\
q_{G}
\end{array}\right]=\left[\begin{array}{c}
\bar{I} \\
U_{G}
\end{array}\right]
$$

where both $E_{Q}$ and $E_{V}$ are $2 N \times M$ sparse matrices, each column of $E_{Q}$ is of the form of $E_{Q m}$, and each column of $E_{V}$ the form of $E_{V m}, q_{G}$ is an $M$ vector formed by terms similar to $q_{G m}$; and $U_{G}$ is an $M$ vector formed by terms similar to $U_{G m}$ in Eq.(15).

Eq.(17) is the modified formulation of Eq.(4). It is a straightforward extension of conventional network equation. From Eq.(17), the reactive powers of PV-buses are calculated together with unknown voltages simultaneously. The detailed solution techniques of Eq.(17) will be discussed in the next section.

4 Solution Techniques and Computational Procedures

\subsection{Solution Techniques}

Eq. (17) is an extension of Eq.(4) with $M$ augmented sparse columns and rows. The solution burden for Eq.(17) is only a little more than that for Eq.(4). By using the constant factors of Eq.(6), the following factorization of the coefficient matrix of Eq.(17) can be derived:

$$
\left[\begin{array}{cc}
\bar{Y} & E_{Q} \\
E_{V}^{T} & 0
\end{array}\right]=\left[\begin{array}{cc}
L & 0 \\
L_{V}^{T} & I
\end{array}\right]\left[\begin{array}{cc}
D & 0 \\
0 & H
\end{array}\right]\left[\begin{array}{cc}
L^{T} & L_{Q} \\
0 & I
\end{array}\right]
$$

where $I$ represents identity matrix, and we have

$$
\begin{array}{ll}
\bar{Y}=L D L & \\
E_{Q}=L D L_{Q} \quad \text { or } & L_{Q}=D^{-1} L^{-1} E_{Q} \\
E_{V}=L D L_{V} \text { or } & L_{V}=D^{-1} L^{-1} E_{V} \\
H=-L_{Q}^{T} D L_{V} &
\end{array}
$$


In Eq.(19) both $L_{Q}$ and $L_{V}$ can be formed by fast forward substitution [9][10] of factors of $\bar{Y}$, in consideration of the sparsity of $E_{Q}$ and $E_{V}$. Matrix $H$ is a full matrix of dimension $M \times M$. Because the number of PV-buses are very limited in a distribution system, $H$ can also be calculated and factorized efficiently, and factorization of Eq.(19) involves a little more additional calculation compared with factorization of Eq.(6).

\subsection{Computational Procedures}

With above factorization, the computational procedures of the proposed method can be briefly described in the following steps.

Step 1 Form the bus admittance matrix $\bar{Y}$, calculate and store its factors by Eq.(6). Assign initial values for bus voltages $V^{(0)}=e^{(0)}+j f^{(0)}$. Set the iteration number $k=0$ and convergence tolerances $\epsilon$.

Step 2 Form the factorization of the coefficient matrix of Eq.(18) by Eq.(19).

Step 3 Compute the right-hand-side vector of Eq.(17) using current voltage vector $V^{(k)}$

Step 4 Perform forward and backward substitution to get the new voltage $V^{(k+1)}$ and reactive power generation increment $\Delta Q_{G}$.

Step 5 Check for convergence: if $\left\|V^{(k+1)}-V^{(k)}\right\| \leq \epsilon$, exit; if not converged, set $k=k+1$ and go to Step 2 .

Computational saving is still available in Step 2, which will be described in the following subsection.

\subsection{Further Developments for Efficiency}

It should be noted that in Step 2, factorization for extended submatrices $E_{Q}$ and $E_{V}$ is performed every iteration, because the sparse elements of $E_{Q}$ and $E_{V}$ are functions of voltages which changes for every iteration. After a few iterations, $E_{Q}$ and $E_{V}$ tend to be invariant and former factors can be reapplied to avoid refactorization. This saving in computation can be achieved by minor modification to Eq.(17). Submatrix $E_{Q}$ can be kept as a constant matrix $E_{Q}^{c}$ without destroying the accuracy of results after convergence, because this submatrix only corresponds to increment variable $\Delta Q_{G}$. But if submatrix $E_{V}$ is kept constant, the derivation of Eq.(17) must be modified. The main idea is to update right-hand-side terms to delete the errors introduced by keeping $E_{V}$ constant. For $k$-th iteration, Eq.(14) can be rewritten as:

$$
e_{m}^{c} \Delta e_{m}^{(k+1)}+f_{m}^{c} \Delta f_{m}^{(k+1)}=\frac{1}{2}\left(\left|V_{m}\right|^{2}-e_{m}^{(k) 2}-f_{m}^{(k) 2}\right)
$$

Substitute $\Delta e_{m}^{(k+1)}=e_{m}^{(k+1)}-e_{m}^{(k)}$ and $\Delta f_{m}^{(k+1)}=f_{m}^{(k+1)}$ $f_{m}^{(k)}$ into the above equation, Eq.(15) can be replaced by:

$$
e_{m}^{c} e_{m}^{(k+1)}+f_{m}^{c} f_{m}^{(k+1)}=U_{G m}^{\prime}
$$

where

$$
U_{G m}^{\prime}=\frac{1}{2}\left(\left|V_{m}\right|^{2}-e_{m}^{(k) 2}-f_{m}^{(k) 2}\right)+e_{m}^{c} e_{m}^{(k)}+f_{m}^{c} f_{m}^{(k)}
$$

From the above equations, it can be seen that only terms of $U_{G m}$ need to be modified as $U_{G m}^{\prime}$. Calculation of other terms need not been changed. The major purpose of this modification is to reflect the influence of the constant submatrix $E_{V}^{c}$ upon the right-hand-side. Generally, after two or three iterations, the submatrices $E_{Q}$ and $E_{V}$ can be treated as constant matrices, therefore Step 2 of the above computational procedure can be skipped to save computing time.

\section{Numerical Examples}

A program developed in Fortran 77 programming language is used to validate the effectiveness of the improved power flow method proposed in this paper. The program was run in an IBM-PC486 (CPU $33 \mathrm{MHz}$ ) computer with NDPFORTRAN compiler. Comparison with Newton's method are also provided. Table 1 lists the main characteristics of test systems, including transmission and distribution systems. Test results, including CPU run-time in seconds and iteration numbers, are listed in Table 2.

Table 1. Characteristics of Test Systems

\begin{tabular}{|c|c|c|c|c|}
\hline $\begin{array}{c}\text { System } \\
\text { Code }\end{array}$ & $\begin{array}{c}\text { No. of } \\
\text { Buses }\end{array}$ & $\begin{array}{c}\text { No. of } \\
\text { Branches }\end{array}$ & $\begin{array}{c}\text { No. of } \\
\text { PV-buses }\end{array}$ & $\begin{array}{c}\text { System } \\
\text { Type }\end{array}$ \\
\hline A & 44 & 51 & 6 & Distribution \\
\hline B & 70 & 74 & 3 & Distribution \\
\hline C & 368 & 384 & 5 & Distribution \\
\hline D & 30 & 41 & 6 & Transmission \\
\hline E & 233 & 341 & 10 & Transmission \\
\hline F & 372 & 536 & 24 & Transmission \\
\hline G & 521 & 725 & 26 & Transmission \\
\hline
\end{tabular}

Table 2. Program Performance for the Test Systems

\begin{tabular}{|c|c|c|c|c|c|c|}
\hline \multirow{2}{*}{$\begin{array}{c}\text { System } \\
\text { Code }\end{array}$} & \multicolumn{2}{|c|}{ Proposed } & \multicolumn{2}{c|}{ Newton's } & \multicolumn{2}{c|}{ Implicit $Z$} \\
\cline { 2 - 7 } & Iter. & CPU & Iter. & CPU & Iter. & CPU \\
\hline A & 6 & 0.11 & 4 & 0.25 & 17 & 0.34 \\
\hline B & 5 & 0.19 & 4 & 0.37 & 11 & 0.51 \\
\hline C & 7 & 1.14 & 5 & 2.04 & 21 & 3.73 \\
\hline D & 5 & 0.09 & 4 & 0.12 & 12 & 0.32 \\
\hline E & 10 & 1.42 & 5 & 2.29 & Div & - \\
\hline F & 14 & 3.96 & 5 & 3.44 & Div & - \\
\hline G & 21 & 6.38 & 6 & 4.37 & Div & - \\
\hline
\end{tabular}

Results in Table 2 indicates that the proposed method is always faster than the traditional implicit $Z$ matrix method no matter what kinds of systems are concerned. The computational time is also less than that for Newton's method for distribution systems. The reason is that each iteration of the proposed method is very fast, it involves no large matrix factorization during iteration process, it also need not to spend time for power mismatch calculation. However, for transmission systems, the convergence of the proposed method becomes poor compared with Newton's method. The robustness of Newton's method is not affected by the system types, which shows its general applicability to power flow studies. For systems whose numbers of PV-buses are relatively large, the computational time of the proposed method increases. Therefore, it can be concluded from Table 2 that the improved implicit $Z$ matrix method is most suitable to distribution systems with a few PV-buses.

Table 3. Robustness Test for System C

\begin{tabular}{|c|c|c|c|c|c|c|}
\hline$|R / X|$ & \multicolumn{2}{|c|}{ Proposed } & \multicolumn{2}{c|}{ Newton's } & \multicolumn{2}{c|}{ FDPF } \\
\cline { 2 - 7 } Ratio & Iter. & CPU & Iter. & CPU & Iter. & CPU \\
\hline $2.0^{*} \mathrm{R}$ & 8 & 1.26 & 5 & 2.13 & $9 / 8$ & 1.31 \\
\hline $3.0^{*} \mathrm{R}$ & 8 & 1.29 & 5 & 2.09 & $10 / 9$ & 1.46 \\
\hline $4.0^{*} \mathrm{R}$ & 9 & 1.48 & 5 & 2.14 & $15 / 14$ & 1.87 \\
\hline $5.0^{*} \mathrm{R}$ & 12 & 1.79 & 6 & 2.32 & Div & - \\
\hline $0.5^{*} \mathrm{X}$ & 9 & 1.42 & 5 & 2.07 & $11 / 10$ & 1.66 \\
\hline $0.35^{*} \mathrm{X}$ & 13 & 1.97 & 7 & 2.53 & $19 / 18$ & 2.10 \\
\hline $0.2^{*} \mathrm{X}$ & Div & - & 9 & 2.84 & Div & - \\
\hline
\end{tabular}


For testing the robustness of the proposed method, some special cases of System $\mathrm{C}$ are given In Table 3 . The tests are performed by changing the $|r / x|$ ratios to create illconditional systems. The first column of Table 3 lists the multipliers used to change the branches' resistances or reactances. For example, $2.0^{*} \mathrm{R}$ means that all the branches' resistances are multiplied by 2.0 and the reactances are kept unchanged. While $0.5^{*} \mathrm{X}$ means that all the branches' reactances are multiplied by 0.5 and all the resistances are kept as original values. It can be seen that Newton's method is most robust. The proposed method converges in most cases, and shows fast computational speed and numerical robustness compared with the traditional FDPF method. The purpose of developing the proposed method is not to replace the Newton's or FDPF methods, but to provide an alternative for certain special application cases, especially for complex network structure analysis of advanced future distribution systems such as FRIENDS.

\section{Conclusions}

For optimal structure analysis of an advanced distribution system like FRIENDS, a great deal of power flow computations are required. The system structure generally changes a little from case to case, with trivial and frequent network operation, a power flow program suitable for this circumstance should be investigated. This paper proposes a modification to conventional implicit $Z$ matrix method for distribution power flow computations. By taking full advantages of the inherent characteristics of distribution systems, improvements can be made in implicit $Z$ matrix method for distribution power flow computations. The proposed method is particularly suitable for large distribution systems, where the number of PV-buses is much smaller compared with the system size. Numerical results indicate that the method is faster than Newton's method and traditional implicit $Z$ matrix method for real world distribution systems. Another salient attribute of the method is that it make structure analysis of the distribution network easier and simpler, which is generally the case in the research of FRIENDS. The method can also be recommended for other power system problems with low level of loads and small number of PV-buses. Further research direction will be towards development of a three phase power flow program based on the proposed method.

(Manuscript received July 28, 1997, revised December 4, 1997)

\section{References}

[1] B. Stott, "Review of Load Flow Calculation Methods," Proceedings of the IEEE, Vol.62, No.7, July 1974, pp.916-929.

[2] W. F. Tinney and C. E. Hart, "Power Flow Solution by Newton's Method", IEEE Trans. on Power App. Syst., Vol.PAS-86, November 1967, pp.1449-1460.

[3] B. Stott and O. Alsac, "Fast Decoupled Load Flow", IEEE Trans. on Power App. Syst., Vol.PAS-93, No.3, May/June 1974, pp.859-869.

[4] D. Shirmohammadi, et al., "A Compensation-based Power Flow Method for Weakly Meshed Distribution and Transmission Networks," IEEE Trans. on PWRS, Vol.PWRS-3, No.2, May 1988, pp.753-762.
[5] G.X. Luo and A. Semlyen, "Efficient Load Flow for Large Weakly Meshed Networks," IEEE Trans. on PWRS, Vol.PWRS-5, No.4, May 1990, pp.1309-13

[6] C.S. Cheng and D. Shirmohammadi, "A Three-Phase Power Flow Method for Real-Time Distribution System Analysis," IEEE Trans. on PWRS, Vol.PWRS-10, No.2, May 1995, pp.671-679.

[7] K. Nara and J. Hasegawa, "A New Flexible, Reliable and Intelligent Electrical Energy Delivery System ," Trans. IEE of Japan, Vol.117-B, No.1, January 1997, pp.47-53.

[8] Y.P. Dusonchet, et al., "Load Flows Using a Combination of Point Jacobi and Newton's Methods," IEEE Trans. on PAS, Vol.PAS-90, May/June 1971, pp.941949.

[9] W. F. Tinney, V. Brandwajn and S. M. Chan, "Sparse Vector Methods", IEEE Trans. Power App. Syst., Vol.PAS-104, No.2, pp.295-301, February 1985.

[10] S. M. Chan and V. Brandwajn, "Partial Matrix Refactorization", IEEE Trans. Power Syst., Vol.PWRS-1, No.1, pp.193-200, February 1986.

\section{Biographies}

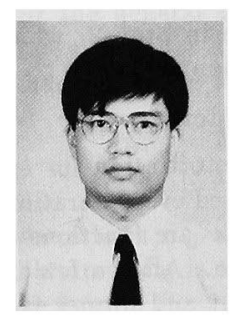

Zheng Yan (Non Member) He received his B.E., M.E. and Ph.D. degrees; all in Electrical Engineering, from Shanghai Jiaotong University, China in 1984, and Tsinghua University, China in 1987 and 1991, respectively. He was a research associate at Institute of Systems Science, Academia Sinica from 1991. He was on research attachment with the Department of Systems Engineering at Ibaraki University, Japan since January 1995.

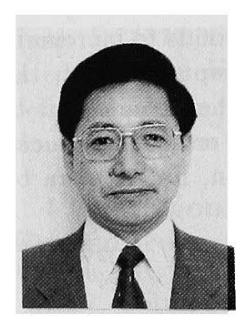

Koichi Nara (Member) He received his B.E., M.E. and Ph.D. degrees from Hokkaido University, Japan, in 1968, 1970 and 1986 respectively. After his industrial and academic experiences in Mitsubishi Electric Co. (1970-1977), Kitami Institute of Technology (1977-1987) and Hiroshima University (1987-1992), he is currently a professor of Department of Systems Engineering at Ibaraki University.

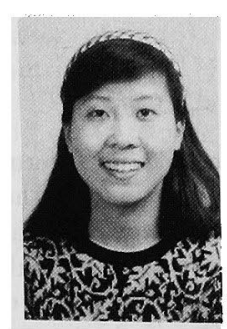

Hongyu Zhou (Non Member) She received her B.E. and M.E. degrees from Tsinghua University, China in 1989 and 1992, respectively. She joined Tsinghua Unisplendour Group of Tsinghua University since 1992 as an Electrical and Electronic Engineer. 